\title{
Serratierte Läsionen im Kolorektum - ein pathologisches Problem oder ein Problem der Pathologen?
}

\author{
Gustavo B. Baretton Daniela E. Aust \\ Institut für Pathologie, Universitätsklinikum «Carl Gustav Carus», Technische Universität Dresden, Deutschland
}

\section{Schlüsselwörter}

Sessiles serratiertes Adenom - Traditionelles serratiertes Adenom - Serratiertes Adenokarzinom .

Serratierter Signal-/Karzinogeneseweg · Kolorektum

\section{Zusammenfassung}

Serratierte Läsionen im Kolorektum stellen eine Problemzone für Kliniker und Pathologen dar; dies liegt zum einen an der noch nicht allgemein bekannten Nomenklatur, zum anderen an dem noch nicht abschließend geklärten Progressionsrisiko der verschiedenen serratierten Polypen. Mittlerweile gilt als akzeptiert, dass neben der klassischen Adenom-Karzinom-Sequenz ein alternativer serratierter und ein sogenannter gemischter Karzinogeneseweg existiert; diese Karzinogenesewege sind in Bezug auf die präinvasiven Vorstufen, die molekularen Pfade und die Prognose der Karzinome heterogen. Bei den serratierten Adenokarzinomen lassen sich ein Niedrigrisiko-Subtyp (Häufigkeit $<20 \%$, mit meist proximaler Lokalisation, dem sessilen serratierten Adenom als Vorläuferläsion, BRAF-Mutation, hoher Mikrosatelliteninstabilität, CpG-Methylierung/hMLH1-Ausfall und einer 5-Jahres-Überlebensrate von $>70 \%$ ) sowie ein Hochrisiko-Subtyp (Häufigkeit $>80 \%$, mit meist distaler Lokalisation, dem traditionellen serratierten Adenom als Vorläuferläsion, KRAS-Mutation, niedriger Mikrosatelliteninstabilität/mikrosatellitenstabil, CpG-Methylierung/ p53-Akkumulation und einer 5-Jahres-Überlebens-Rate von $<30 \%$ ) unterscheiden. Eine molekularpathologische Bestimmung des Mikrosatellitenstatus und einer BRAFoder KRAS-Mutation in Verbindung mit einer hMLH1und p53-Immunhistochemie lässt eine bessere Unterscheidung dieser beiden Typen zu und wird klinisch zunehmend relevant.

\section{Keywords}

Sessile serrated adenoma - Traditional serrated adenoma - Serrated adenocarcinoma . Serrated pathway . Colorectum

\section{Summary}

Serrated Lesions of the Colorectum - a Pathological or a Pathologist's Problem?

The classification and treatment of serrated lesions in the lower gastrointestinal tract remains a difficult task for clinicians and surgical pathologists; on the one hand, due to the inconsistent nomenclature of serrated lesions in the lower gastrointestinal tract and, on the other hand, due to the poor understanding of the underlying mechanisms and risk factors for progression of these lesions. It is widely accepted that in addition to the classic adenoma-carcinoma sequence a recently discovered serrated or mixed pathway is important in colorectal carcinogenesis. These two pathways are heterogeneous with regard to precursor lesions, molecular mechanisms and prognosis. Depending on their molecular signature, serrated colorectal adenocarcinomas, for instance, can be subdivided into a low-risk (frequency $<20 \%$, often with proximal location, the sessile serrated adenoma as a precursor lesion, BRAF mutation, high microsatellite instability, CpG methylation/hMLH1 loss and a 5-year survival rate of $>70 \%$ ) and a high-risk subtype (frequency $>80 \%$, often with distal location, the traditional serrated adenoma as a precursor lesion, KRAS mutation, low microsatellite instability/microsatellite stable, CpG methylation/p53 accumulation and a 5-year survival rate of $<30 \%$ ). The evaluation of the microsatellite status as well as the detection of BRAF or KRAS mutations together with immunohistochemistry for hMLH1 and p53 will allow a discrimination between these two subtypes and will become increasingly important in the future.

\section{KARGER \\ Fax +497614520714 \\ Information@Karger.de}

www.karger.com
(C) 2011 S. Karger GmbH, Freiburg

1662-6664/11/0274-0309\$38.00/0

Accessible online at:

www.karger.com/vim
Prof. Dr. med. habil. Gustavo B. Baretton

Institut für Pathologie

Universitätsklinikum «Carl Gustav Carus», Technische Universität Dresden

Fetscherstraße 74, 01307 Dresden, Deutschland

Tel. +49 351 458-3000, Fax -4328

gustavo.baretton@uniklinikum-dresden.de 


\section{Einleitung}

In den letzten Jahren zeigten molekulare Befunde, dass sich das kolorektale Karzinom (KRK) über verschiedene Karzinogenesewege entwickeln kann. Neben der 1989 von Fearon und Vogelstein [1] beschriebenen klassischen Adenom-Karzinom-Sequenz stellt der sogenannte serratierte Karzinogeneseweg inzwischen einen akzeptierten alternativen Karzinogeneseweg dar [2].

Bereits 1999 konnten Iino und Jass [3] zeigen, dass hyperplastische Polypen (HP)/serratierte Polypen der Manifestation mikrosatelliteninstabiler (MSI-positiver) KRK vorausgehen können, was darauf hindeutet, dass serratierte Polypen in die Karzinogenese einer Subgruppe von KRK involviert sind. Das sessile serratierte Adenom (SSA) und das traditionelle serratierte Adenom (TSA) werden heute als präkanzeröse Läsionen des sogenannten serratierten Adenokarzinoms angesehen; dabei handelt es sich um einen distinkten Subtyp des KRK. Die molekularen Veränderungen des serratierten Karzinoms (BRAF-Mutation, CIMP-Pathway) passen nicht zu denen der klassischen Adenome. Morphologische und molekulargenetische Charakteristika legen überdies die Existenz von zwei unterschiedlichen serratierten Karzinogenesewegen nahe.

Die Kenntnis und richtige diagnostische Einordnung der präinvasiven und invasiven Läsionen des serratierten Karzinogenesewegs ist sowohl bedeutsam für die Überwachung der Patienten mit Vorläuferläsionen (Polypen-Management) als auch für eine differenzierte Therapie der Patienten mit serratierten KRK sowie zukünftig eventuell auch für eine Karzinomprävention.

\section{Pathogenese}

Im Gegensatz zu der klassischen Adenom-KarzinomSequenz, bei der durch schrittweise Akkumulation von Tumor-Suppressorgen-Verlusten (z.B. APC, p53) und OnkogenZugewinnen (z.B. KRAS) eine chromosomale Instabilität (CIN) mit Aneuploidie entsteht [4], kommt es beim serratierten Karzinogeneseweg zunächst zu einer Störung der physiologischen Apoptose in den Krypten. Die charakteristische serratierte Kryptenmorphologie lässt sich aus einem «Aufstau» einer erhöhten Zahl retinierter Zellen bei dieser Apoptosestörung erklären [5]; dieser Prozess wird durch aktivierende Mutationen im BRAF- oder KRAS-Gen vermittelt (sogenannte Onkogen-induzierte Seneszenz, OIS), d.h. eine durch Onkogen-Aktivierung ausgelöste initiale Epithel-Proliferation wird durch Induktion einer Zellzyklusblockade (z.B. via p16) gegenreguliert [6, 7]. Durch die resultierende verlängerte Lebenszeit der Kryptenepithelien kann es im Rahmen des Überalterungsprozesses (Seneszenz) zu einer vermehrten Methylierung der DNA kommen - speziell im Bereich sogenannter CpG-Inseln in Gen-Promotoren, die als molekulare

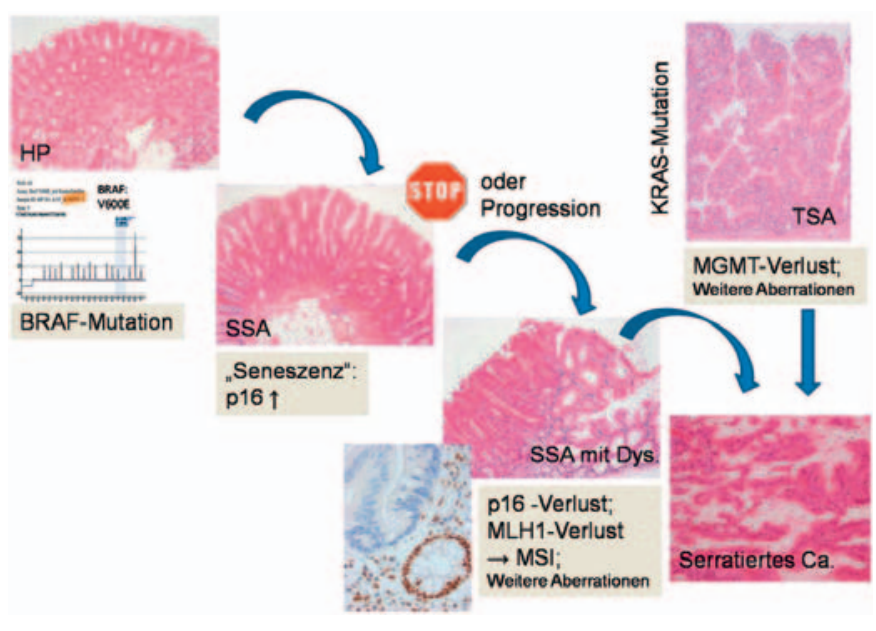

Abb. 1. Progressionsmodell serratierter Adenome im Kolorektum [25].

«Schalter» für die Transkriptionsregulation dienen. Dadurch kommt es zum «Abschalten» (sogenanntes Silencing) der Transkription der betroffenen Gene [8], z.B. von ZellzyklusKontroll-Genen wie p16 und/oder von Reparaturgenen (z.B. $h M L H 1, M G M T)$, was wiederum zu Mikrosatelliteninstabilität (MSI) führt: Man spricht daher auch vom CIMP(CpGIsland-Methylation)-Phänotyp; ähnlich wie bei der MSI lassen sich ein hoher (CIMP-H) und ein niedriger (CIMP-L) Methylierungstyp unterscheiden (Abb. 1).

Übereinstimmende genetische Veränderungen wurden in bestimmten serratierten Polypen, nicht jedoch in «konventionellen» Adenomen nachgewiesen: in TSA (primär KRASMutation; sekundär CIMP und Mutationen von $p 53, A P C$ ) und in SSA (primär BRAF-Mutation; sekundär CIMP mit hMLH1-Ausfall). Welche Faktoren die Methylierung und damit die Progression der SSA und TSA beeinflussen, ist bislang unklar; neben einer individuell unterschiedlichen genetischen Prädisposition werden unter anderem ein hoher BMI, Rauchen und Östrogen-Entzug als Risikofaktoren für serratierte Polypen und Karzinome diskutiert (Übersicht siehe [2]).

Das serratierte Adenokarzinom ist ein distinkter Subtyp des KRK, der 1992 von Jass und Smith [9] erstmals erwähnt und im Jahr 2000 von Yao [10] primär beschrieben wurde. Mindestens 7,5\% aller KRK und etwa 17,5\% der rechtsseitigen KRK zeigen einen serratierten Phänotyp; histologisch lassen sich verschiedene Wachstumsformen (serratiert, muzinös und trabekulär) unterscheiden [2]. Die molekularen Veränderungen des serratierten Karzinoms stützen das Konzept eigenständiger präneoplastischer Läsionen, da klassische Adenome diese molekularen Veränderungen nicht bzw. nur selten aufweisen. Morphologische und molekulargenetische Charakteristika legen dabei die Existenz von zwei unterschiedlichen serratierten Karzinogenesewegen dar [2]:

- den MSI-H(hohe Mikrosatelliteninstabilität)-serratierten Weg, der vom SSA als präkanzeröser Läsion ausgeht (möglicherweise über den Zwischenschritt eines «SSA mit 
intraepithelialer Neoplasie»/«gemischten Polypen»). Die SSA und Karzinome dieses Wegs weisen häufig eine hMLH1-Promotormethylierung auf, die zum MSI-H-Phänotyp führt; diese serratierten Karzinome sind der Prototyp der sporadischen MSI-H-KRK, meist proximal lokalisiert und klinisch mit einer günstigeren Prognose assoziiert (sogenannte alternative serratierte Route [11] bzw. molekularer Typ 1 nach Jass [4]).

- den MSI-L(niedrige Mikrosatelliteninstabilität)- oder MSS (mikrosatellitenstabil)-serratierten Weg, der vom TSA ausgeht. Dieser Pfad ist durch einen MSI-L- oder MSSPhänotyp charakterisiert, kann aber auch geringe chromosomale Instabilität und vermehrte Methylierung zeigen; diese Tumoren sind überwiegend im distalen Kolon und Rektum lokalisiert und weisen klinisch eine ungünstigere Prognose auf (sogenannter Mischtyp bzw. molekularer Typ 3 nach Jass [12]).

\section{Nomenklatur präinvasiver Neoplasien im}

\section{Gastrointestinaltrakt: Aktualisierte WHO-Klassifikation} 2010

Leider trägt die Terminologie in der kürzlich aktualisierten WHO-Klassifikation (4. Auflage, November 2010) [13] mehr zur Verwirrung als zum besseren Verständnis der präinvasiven Neoplasien im Gastrointestinaltrakt bei. Wurde im Jahr 2000 in der dritten Auflage der Begriff «Dysplasie» durch den der «Intraepithelialen Neoplasie» ersetzt, wird der Terminus «Dysplasie» jetzt wiederbelebt und, was gravierender ist, eine partielle, aber nicht konsistente Vermischung dieser Begrifflichkeiten vorgenommen.

Aktuell werden von der WHO zwei Termini verwendet:

1. die intraepitheliale Neoplasie (IEN) als Begriff für nicht invasive Läsionen, die ganz allgemein zytologische oder architektonische Störungen aufweisen, die als Ausdruck zugrunde liegender molekularer Aberrationen gewertet werden können und ein Progressionspotenzial aufweisen. Subsumiert sind somit morphologisch erkennbare Läsionen mit (z.B. tubuläres Adenom) oder ohne zytologische Atypie/Dysplasie (z.B. SSA); die IEN-Typen und ihre assoziierten morphologischen Charakteristika unterscheiden sich von Organ zu Organ.

2. die Dysplasie als «histologisch eindeutig neoplastisches Epithel ohne Anhalt für ein invasives Wachstum»; der Begriff «Dysplasie» unterscheidet sich von der IEN somit durch das Vorliegen morphologischer/zytologischer Neoplasie-Zeichen.

Zur Begründung, dass man sich bei der Neuauflage der WHO-Klassifikation nicht auf einen einzelnen Begriff für nicht invasive Neoplasien einigen konnte, wird angeführt, dass

- spezifische zytologische und/oder architektonische Störungen von Vorläuferläsionen sich entsprechend ihrer anato-
Abb. 2. HP mit Serratierung im oberen Kryptendrittel, schmaler Kryptenbasis und basalständigen kleinen Zellkernen (Vergrößerung $\times 10$ ).

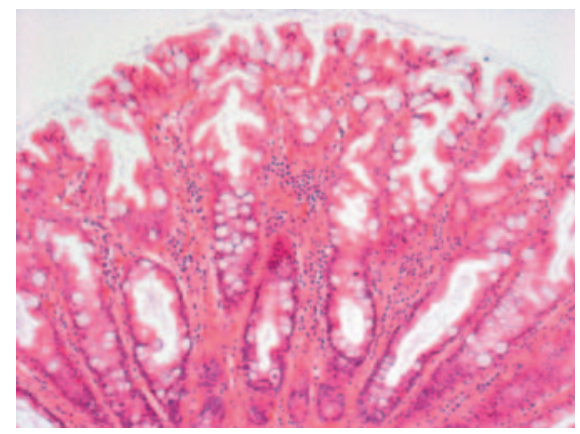

mischen Lokalisation (Organ) erheblich unterscheiden können, ebenso wie das ortständige Epithel in seinen biologischen Eigenschaften und in seinem malignen Potenzial wesentliche Unterschiede von Organ zu Organ aufweist;

- aktuelle Fortschritte in unserem Verständnis des Zeitpunkts, des Typs und der Abfolge molekularer Veränderungen, die zur neoplastischen Transformation führen, gezeigt haben, dass klonale molekulare Aberrationen, die zur Dysregulation der Zellproliferation/-differenzierung und zu einem erhöhten Risiko einer weiteren neoplastischen Progression führen, bereits ohne die typischen morphologischen Merkmale einer nicht invasiven Neoplasie auftreten können (z.B. Aneuploidie, TP53- und CDKN2A-Mutationen bei Barrett-Ösophagus und bei chronisch entzündlichen Darmerkrankungen).

\section{Nomenklatur der serratierten Polypen im Kolorektum}

Um zu einer einheitlichen Terminologie im deutschsprachigen Raum beizutragen und die Kriterien für die histopathologische Diagnostik serratierter Läsionen im Kolorektum [4] klarer herauszuarbeiten, hat die Arbeitsgemeinschaft Gastroenteropathologie der Deutschen Gesellschaft für Pathologie im vergangenen Jahr ein Konsensus-Papier publiziert [14]. Diese Nomenklatur stimmt in weiten Teilen mit der neuen WHO-Klassifizierung überein; kritikwürdig, weil inkonsistent, ist an der WHO-Einteilung, dass das SSA, welches per definitionem einer IEN entspricht (meist ohne, aber gelegentlich auch mit Dysplasie), synonym sessiler serratierter Polyp (SSP) genannt werden darf. Im Folgenden werden die verschiedenen Typen der serratierten Läsionen kurz vorgestellt.

\section{Hyperplastischer Polyp}

Der HP ist die mit Abstand häufigste serratierte Läsion im Kolorektum (80-90\%); HP kommen vorwiegend im linken Kolon und Rektum vor und sind meist $<5 \mathrm{~mm}$ (Abb. 2). Histomorphologische Merkmale sind elongierte Krypten mit serratierter Architektur in der oberen Kryptenhälfte, gelegentlich auch nur im oberen Drittel bzw. nur an der Oberfläche; dies führt zu einer unregelmäßigen Erweiterung mit «gezahnten» Krypten-Lumina in der oberen Hälfte der Mukosa. Die 
Proliferation in der basalen (nicht serratierten) Kryptenhälfte ist regulär. Die Kerne sind klein, uniform und basalständig; es findet sich keine Kernschichtung in der oberen Kryptenhälfte. Eine zytologische Dysplasie bzw. eine IEN fehlen obligat; auch liegt (im Gegensatz zum SSA) keine strukturelle bzw. architektonische Atypie vor.

In der Literatur werden entsprechend der zytoplasmatischen Differenzierung drei HP-Subtypen unterschieden:

1. der mikrovesikuläre Typ (am häufigsten, oftmals mit BRAF-Mutation),

2. der becherzellreiche Typ (häufig mit KRAS-Mutation) und

3. der muzinarme Typ (seltenste Variante).

Da es in der Praxis sehr schwierig ist, diese Subtypen zu differenzieren, und die klinische Bedeutung bislang zweifelhaft ist, wird 2010 auch von der WHO empfohlen, derzeit in der Routinediagnostik keine Subklassifikation der HP vorzunehmen. Das neoplastische Progressionsrisiko für die überwiegende Mehrzahl der kleinen und distal im Kolon sowie im Rektum lokalisierten HP ist sehr gering. Allerdings sollten HP mit einem Durchmesser von $>10 \mathrm{~mm}$ und einer rechtskolischen Lokalisation abgetragen werden, da einzelne Fallberichte und kleine Fallserien über Adenokarzinome in Assoziation mit «großen» oder «Riesen»-HP (meist definiert als >10 mm) nahe legen, dass zumindest ein Teil der HP ein malignes Potenzial besitzt.

Das Auftreten multipler HP in Form des Syndroms der serratierten Polypose (früher: hyperplastische Polypose) ist eindeutig mit dem Auftreten von KRK assoziiert; genetischer Hintergrund und Pathogenese der serratierten Polypose sind bislang jedoch unbekannt. Die Definition der serratierten Polypose nach WHO 2010 [13] lautet:

1. mindestens 5 histologisch bestätigte serratierte Polypen proximal des Sigmas, davon zwei $>1 \mathrm{~cm}$, oder

2. jede Zahl serratierter Polypen proximal des Sigmas bei Patienten mit einem/einer erstgradig Verwandten mit hyperplastischer Polypose, oder

3. mehr als 20 serratierte Polypen jeglicher Größe im Kolon verteilt.

\section{Sessiles serratiertes Adenom}

Der zweithäufigste serratierte Polypen-Typ ist das SSA (ICD-O Code: 8213/0; nach WHO 2010 synonym auch SSP) mit einer Häufigkeit von ca. 15-20\%. Wie bereits aus dem Namen hervorgeht, handelt es sich dabei um eine nicht polypoide Veränderung, die typischerweise mehr als $5 \mathrm{~mm}$ groß und rechtskolisch lokalisiert ist [15].

Die histomorphologischen Charakteristika des SSA sind Hyperserratierung und Dilatation der Krypten (mit Reduktion des Stromas bzw. Verschiebung des Epithel-/StromaVerhältnisses durch Rücken-an-Rücken-Lagerung dilatierter Krypten), oft bis an die Basis mit T- und L-förmigen Verzweigungen; die Lamina muscularis mucosae erscheint häufig ausgedünnt, zudem können Krypten unterhalb der L. muscularis mucosae vorkommen (sogenannte Mikroherniation). Weitere

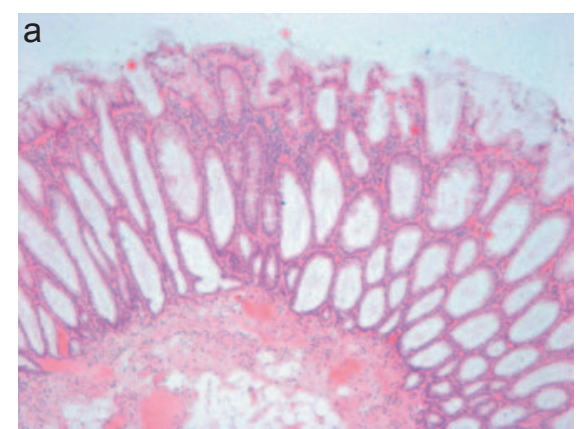

Abb. 3a. SSA mit säulenförmig dilatierten Krypten und T-förmiger Verzweigung an der Kryptenbasis (Vergrößerung $\times 4)$. b SSA mit basal verzweigten Krypten (T- und L-förmig) und Becherzellen an der Kryptenbasis (Vergrößerung $\times 10$ ).

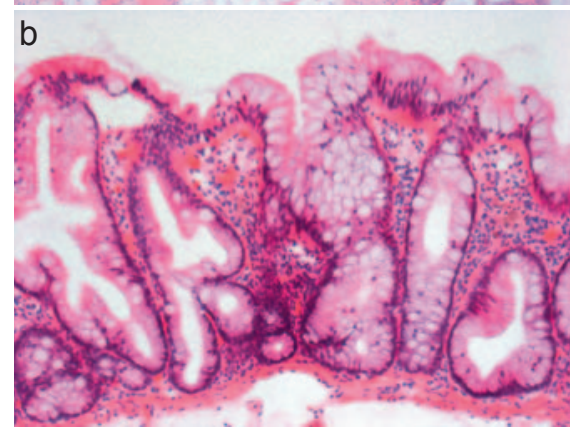

Merkmale des SSA sind das Vorkommen reifer Becherzellen an der Kryptenbasis, Verschiebung der Proliferationszone ins mittlere Kryptendrittel und der Nachweis von gering vergrößerten vesikulären Zellkernen mit Nukleolen (Abb. 3). Die vier nach Konsensus der Arbeitsgemeinschaft Gastrointestinale Pathologie der Deutschen Gesellschaft für Pathologie diagnostisch entscheidenden Kriterien sind:

- Hyperserratierung/Serratierung im unteren Kryptendrittel (mit oder ohne Verzweigung)

- T- und L-förmige Krypten oberhalb der L. muscularis mucosae

- «invertierte» Krypten (Mikroherniation) unterhalb der L. muscularis mucosae

- «säulenförmige» Dilatation im unteren Kryptendrittel (mit oder ohne Schleimfüllung)

Mindestens zwei der vier genannten Kriterien in mindestens 2 Krypten, die nicht nebeneinander liegen müssen, sollten zutreffen. Die genannten diagnostischen Kriterien gelten auch für Läsionen der Appendix vermiformis.

Da für die SSA-Diagnose die Erfassung der basalen Schleimhautanteile essenziell ist, wird empfohlen, bei zweifelhaften Fällen (z.B. bei unzureichender Biopsiegröße mit nur oberflächlich erfassten Anteilen) den Terminus SSP zu verwenden und verbal darauf hinzuweisen, dass eine sichere Differenzierung zwischen einem HP und einem SSA am vorliegenden Gewebsmaterial nicht möglich ist [14].

Eine besondere Problemzone in der Diagnostik und Nomenklatur stellen die SSA dar, die eine IEN aufweisen. Aktuell wird von der «Adenom-artigen» IEN eine zytologische serratierte Dysplasie («serrated type») abgegrenzt, die irregulär (meist «bottom-up») in den polypösen Läsionen auftritt; in der internationalen Literatur wird bei Nachweis solcher dys- 


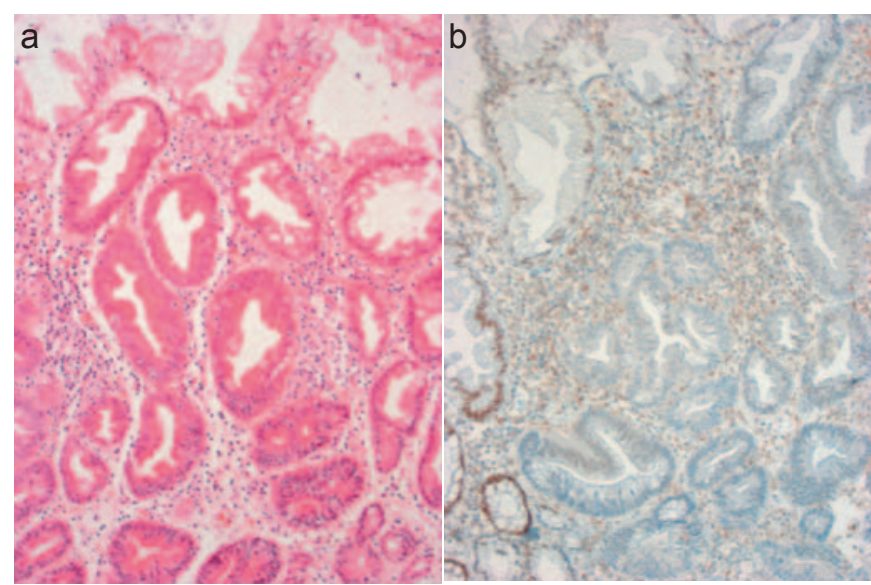

Abb. 4a. SSA mit serratierter, von der Kryptenbasis ausgehender, zytologischer Dysplasie mit Stratifizierung der Zellkerne und zugunsten der Kerne verschobener Kern-Plasma-Relation (Vergrößerung $\times 10$ ). b Immunhistologischer Nachweis des Expressionsverlusts von hMLH1 in den zytologisch dysplastischen Arealen des SSA als histomorphologisches Korrelat der Promotorhypermethylierung des $h M L H 1-G e n s$ (Vergrößerung $\times 10$ ).

plastischer Veränderungen in einem SSA zum Teil von einem gemischten Polyp («mixed polyp») gesprochen. Da dies möglicherweise zu einer unzulässigen Vermischung von Entitäten führt bzw. das maligne Potenzial von SSA mit serratierter Dysplasie außer Acht lässt, soll diese Gruppe provisorisch als «SSA mit Dysplasie/IEN» (mit Angabe hochgradig oder geringgradig) klassifiziert werden, um der möglicherweise besonderen biologischen Bedeutung dieser Läsionen Rechnung zu tragen (Abb. 4).

Die gemischten Polypen wurden ursprünglich als Kollisionstumoren interpretiert; dabei liegen typischerweise Mischformen aus «klassischen» Adenomen mit unterschiedlichem (adenomatösem) Dysplasie-/IEN-Grad und serratierten Läsionen vor. Diese Bezeichnung ist nicht optimal, da sie den präinvasiven Charakter der Läsionen nicht adäquat zum Ausdruck bringt, sollte aber in Anlehnung an die internationale Nomenklatur verwendet werden; allerdings sollen nach dem deutschen Konsensus-Papier [14] zunächst die Bestandteile des gemischten Polypen genannt und der Begriff «gemischter Polyp» erst dahinter in Klammern geführt werden (z.B. «sessiles serratiertes und tubulovillöses Adenom (sogenannter gemischter Polyp) mit geringgradiger intraepithelialer Neoplasie/Dysplasie»)).

\section{Traditionelles serratiertes Adenom}

Das TSA (ICD-O Code 8213/0) stellt die seltenste Variante der serratierten Veränderungen dar (1-6\%); es wurde bereits 1990 mit der Bezeichnung «Serrated Adenoma» als eine seltene Adenomvariante (ca. 1\%) beschrieben. Klinisch handelt es sich beim TSA um gestielte oder (tubulo-)villöse Polypen, die häufiger im linken $(60 \%)$ als im rechten Kolon bei zumeist älteren Patienten auftreten [15].
Abb. 5a. Ausschnitt aus einem TSA mit geringgradiger IEN/ Epitheldysplasie mit prominenter Zytoplasmaeosinophilie und gleichmäßigen stäbchenförmigen Zellkernen (Vergrößerung $\times 10)$. b Ausschnitt aus einem TSA mit geringgradiger IEN/Epitheldysplasie mit sogenannten ektopen Kryptenformationen (Vergrößerung $\times 20$ ).

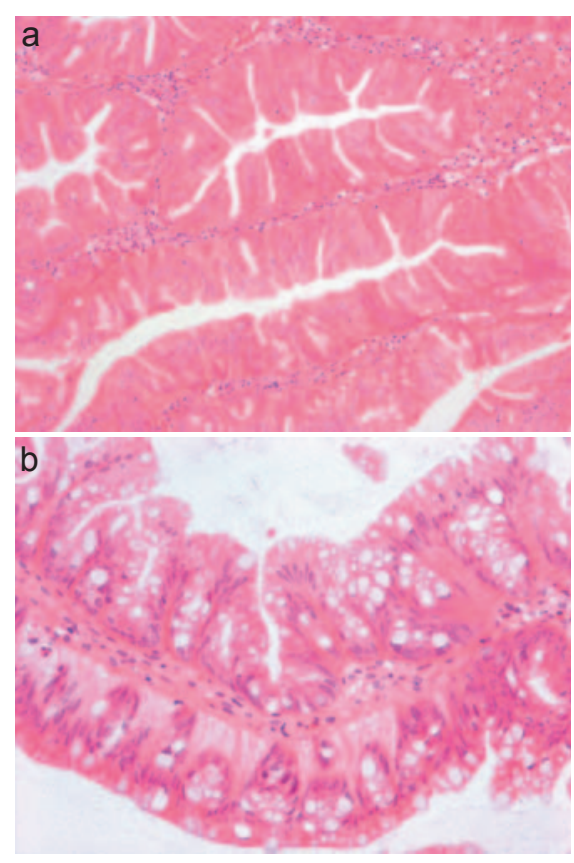

Das TSA zeigt histologisch definitionsgemäß dysplastische Epithelveränderungen im Sinne einer IEN unterschiedlichen Schweregrades (in ca. $90 \%$ eine geringgradige IEN oder in ca. $10 \%$ eine hochgradige IEN). Überdies liegen eine ausgeprägte Serratierung und eine diffuse zytoplasmatische Eosinophilie vor; dabei treten intraepitheliale Mikro-Azini, sogenannte «Ectopic Crypt Formation» (ECF), auf, d.h. Krypten, die keinen Bezug zur L. muscularis mucosae haben, sondern seitlich aus anderen Krypten «aussprossen» (Abb. 5).

Filiforme serratierte Adenome mit fingerförmiger Proliferation und prominenter Serratierung sind eine ungewöhnliche TSA-Variante (mit vergleichbarem Immunphänotyp für Methylguanin-DNA-Methyltransferase (MGMT), MMR-Proteine, $\beta$-Catenin, Ki-67 und p53), die bevorzugt im Rektum vorkommen.

\section{Krebsrisiko}

Von Morson [16] wurden die epithelialen Polypen im Kolorektum dogmatisch in «nicht neoplastische Läsionen», d.h. die hyperplastischen (metaplastischen) Polypen, und die «neoplastischen» Adenome (tubulär, tubulo-villös und villös) unterteilt; das Unterscheidungskriterium war der Nachweis zytologischer Atypien/Dysplasien, der für die Adenom-Diagnose als conditio sine qua non angesehen wurde. Während so die Adenome als «Präneoplasien» klassifiziert wurden, mit der klinischen Implikation, die Läsionen zu entfernen und die Patienten endoskopisch zu überwachen, wurde den HP als Prototyp der serratierten Polypen im Kolorektum kein Entartungspotenzial zugeschrieben. Mittlerweile kann jedoch ein Progressions- bzw. Malignomrisiko für serratierte Adenome 
Tab. 1. Nachsorgeintervalle nach der S3-Leitlinie «Kolorektales Karzinom zum Polypenmanagement» [19]

\begin{tabular}{|c|c|c|}
\hline Typ der Läsion & Erste Kontrolle & Weitere Kontrolle \\
\hline Nicht neoplastische Polypen & keine & keine \\
\hline $\begin{array}{l}\text { Niedrigrisiko-Adenom ( } 1-2 \text { Adenome, jeweils }<1 \mathrm{~cm} \text {, tubulär, } \\
\text { nur niedriggradige Dysplasie) }\end{array}$ & 5 Jahre & 5 Jahre \\
\hline $\begin{array}{l}\text { Hochrisiko-Adenom [26] ( } \geq 3 \text { tubuläre Adenome, } \geq 1 \text { Adenom } \geq 1 \mathrm{~cm} \text {, } \\
\text { Adenom mit villöser Struktur, } \geq 1 \text { Adenom mit hochgradiger } \\
\text { Dyplasie, } \geq 10 \text { Adenome jede Größe/Histologie; SSA, TSA und } \\
\text { gemischte Polypen) }\end{array}$ & $\begin{array}{l}3 \text { Jahre (bei }>10 \text { Adenomen, } \\
\text { eventuell auch kürzer; individuelle } \\
\text { Kriterien/Familienanamnese) }\end{array}$ & $\begin{array}{l}5 \text { Jahre (falls erste } \\
\text { Kontrolle unauffällig) }\end{array}$ \\
\hline $\begin{array}{l}\text { Bei histologisch nicht bestätigter vollständiger Abtragung (auch } \\
\text { wenn makroskopisch komplett) }\end{array}$ & 2-6 Monate & $\begin{array}{l}\text { nach Typ und Befund der } \\
\text { ersten Kontrolle }\end{array}$ \\
\hline $\begin{array}{l}\text { In Piecemeal-Technik abgetragene große, flache oder sessile } \\
\text { Adenome }\end{array}$ & 2-6 Monate & 3 Jahre, dann 5 Jahre \\
\hline $\begin{array}{l}\text { Niedrigrisiko-Karzinom nach kompletter lokaler Entfernung } \\
\text { (inklusive Piecemeal) }\end{array}$ & 6 Monate & 2 Jahre \\
\hline
\end{tabular}
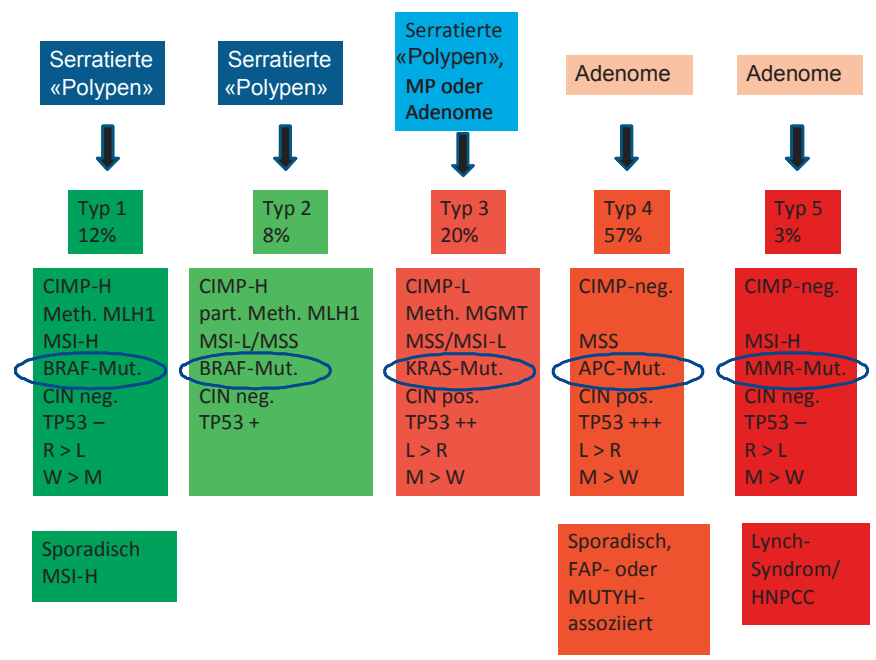

Abb. 6. Die unterschiedlichen Karzinogenesewege des KRK (modifizert nach Jass [4]).

(d.h. SSA und TSA) als gesichert gelten; dies wird durch verschiedene Befunde gestützt [2]:

1. Eine maligne Progression von Veränderungen, die primär als HP klassifiziert wurden.

2. Mit einer hyperplastischen Polypose assoziierte KRK.

3. Der Nachweis von zytologischen Atypien/dysplastischen Veränderungen in TSA und gelegentlich in SSA (sogenannte SSA mit IEN/«gemischter Polyp»).

4. Metachrone KRK und SSA in Assoziation mit SSA oder primär als HP klassifizierten Läsionen.

5. Serratierte Adenomresiduen im Randbereich von KRK.

6. Morphologische und immunhistologische Ähnlichkeiten von bestimmten KRK mit HP und serratierten Adenomen.

7. Übereinstimmende genetische und epigenetische Veränderungen.
8. Übereinstimmende distinkte Expressionsprofile von KRK mit serratierter Morphologie und serratierten Adenomen in der RNA-Array-Analyse.

Vom SSA als präkanzeröser Läsion soll der MSI-H-serratierte Weg ausgehen (möglicherweise über den Zwischenschritt eines SSA mit IEN/gemischten Polypen). Die SSA und Karzinome dieses Wegs weisen häufig eine hMLH1-Promotormethylierung auf, die zum MSI-H-Phänotyp führt; diese serratierten Karzinome sind der Prototyp der sporadischen MSI-H-KRK, meist proximal lokalisiert und klinisch mit einer günstigeren Prognose assoziiert (sogenannte alternative serratierte Route bzw. molekularer Typ 1 nach Jass) [4] (Abb. 6). (Anmerkung: Bei Karzinomen mit BRAF-Mutation, aber ohne MSI-H, d.h. MSI-L oder MSS, scheint jedoch die Prognose besonders ungünstig zu sein.)

Vom TSA soll der MSI-L- oder MSS-serratierte Weg der serratierten Karzinogenese seinen Ausgang nehmen. Dieser Pfad ist durch einen MSI-L- oder MSS-Phänotyp charakterisiert, kann aber auch geringe CIN und vermehrte Methylierung zeigen; die Tumoren sind überwiegend im distalen Kolon und Rektum lokalisiert und weisen klinisch eine ungünstigere Prognose auf (sogenannter Mischtyp bzw. molekularer Typ 3 nach Jass) [4].

Für den Kliniker ist dabei interessant, wie schnell die Progression abläuft und ob der serratierte Karzinogeneseweg zumindest teilweise für die sogenannten Intervallkarzinome (in ca. $1 \%$ treten bei der Polypennachsorge innerhalb von 3 Jahren Karzinome auf) verantwortlich ist [15]. Die Datenlage ist hierfür noch nicht abschließend aussagekräftig. In einer großen retrospektiven US-amerikanischen Studie [17] mit 179111 Koloskopien lag das Durchschnittsalter der Patienten mit SSA bei 61 Jahren, mit SSA mit geringgradiger Dysplasie bei knapp 66 Jahren, bei SSA mit hochgradiger Dyplasie bei 72 Jahren und für SSA mit Karzinom bei 76 
Jahren, sodass eine Progressionszeit von ca. 15 Jahren postuliert wurde; dieses Intervall war in der genannten Untersuchung deutlich länger als für konventionelle tubuläre Adenome (ca. 5 Jahre) und Adenome mit villöser Komponente (ca. 3 Jahre). Eine andere Studie [18] und auch die Daten aus dem eigenen Untersuchungsmaterial (unveröffentlicht) mit allerdings deutlich kleineren Fallzahlen sprechen jedoch für eine schnellere Progression der serratierten als der «klassischen» Adenome. Diese Daten rechtfertigen zumindest das in der deutschen S3-Leitlinie empfohlene Kontrollintervall von 3 Jahren nach kompletter Entfernung eines SSA oder TSA (Tab. 1) [19]).

Inwieweit sich KRK, die über den serratierten oder den Misch-Karzinogeneseweg entstanden sind, hinsichtlich ihres Ansprechens auf die derzeitigen adjuvanten Chemotherapien unterscheiden und ob die spezifischen molekularen Veränderungen dieser Karzinogenesewege einer zielgerichteten («targeted») Therapie (CIMP: demethylierende Agenzien; BRAF: Sorafenib usw.) zugänglich sind [20-22], muss in prospektiven Studien geklärt werden. Als weitgehend sicher kann ein schlechteres Ansprechen auf 5-Fluorouracil-basierte Therapieschemata beim Niedrigrisiko-Subtyp des serratierten Karzinoms gelten [23], ebenso ein fehlendes Ansprechen der Karzinome mit KRAS-Mutationen auf anti-EGFR-Therapie im palliativen Setting [24].

\section{Disclosure Statement}

Keine Interessenkonflikte.

\section{Literatur}

1 Fearon ER, Vogelstein B: A genetic model for colorectal tumorigenesis. Cell 1990;61:759-767.

2 Mäkinen MJ: Colorectal serrated adenocarcinoma. Histopathology 2007;50:131-150.

3 Iino H, Jass JR, Simms LA, Young J, Leggett B, Ajioka Y, Watanabe H: DNA microsatellite instability in hyperplastic polyps, serrated adenomas, and mixed polyps: a mild mutator pathway for colorectal cancer? J Clin Pathol 1999;52:5-9.

4 Jass JR: Classification of colorectal cancer based on correlation of clinical, morphological and molecular features. Histopathology 2007;50:113-130.

5 Tateyama H, Li W, Takahashi E, Miura Y, Sugiura $\mathrm{H}$, Eimoto T: Apoptosis index and apoptosisrelated antigen expression in serrated adenoma of the colorectum: the saw-toothed structure may be related to inhibition of apoptosis. Am J Surg Pathol 2002;26:249-256.

6 Jass JR, Whitehall VL, Young J, Leggett BA: Emerging concepts in colorectal neoplasia. Gastroenterology 2002;123:862-876.

7 Kambara T, Simms LA, Whitehall VL, Spring KJ, Wynter CV, Walsh MD, Barker MA, Arnold S, McGivern A, Matsubara N, Tanaka N, Higuchi T, Young J, Jass JR, Leggett BA: BRAF mutation is associated with DNA methylation in serrated polyps and cancers of the colorectum. Gut 2004; 53:1137-1144.

8 Toyota M, Ahuja N, Ohe-Toyota M, Herman JG, Baylin SB, Issa JP: CpG island methylator phenotype in colorectal cancer. Proc Natl Acad Sci U S A 1999;96:8681-8686.

9 Jass JR, Smith M: Sialic acid and epithelial differentiation in colorectal polyps and cancer - a morphological, mucin and lectin histochemical study. Pathology 1992;24:233-242.

10 Yao T, Nishiyama K, Oya M, Kouzuki T, Kajiwara M, Tsuneyoshi M: Multiple 'serrated adenocarcinomas' of the colon with a cell lineage common to metaplastic polyp and serrated adenoma. Case report of a new subtype of colonic adenocarcinoma with gastric differentiation. J Pathol 2000;190:444-449.
11 Kirchner T, Reu S: Development of molecularpathologic entities of colorectal cancer. Pathologe 2008;29(suppl 2):264-269.

12 Baker K, Zlobec I, Tornillo L, Terracciano L, Jass JR, Lugli A: Differential significance of tumour infiltrating lymphocytes in sporadic mismatch repair deficient versus proficient colorectal cancers: a potential role for dysregulation of the transforming growth factor-beta pathway. Eur J Cancer 2007;43: 624-631.

13 Bosman FT, Carneiro F, Hruban RH, Theise ND (eds): WHO Classification of Tumours of the Digestive System, ed 4. Lyon, IARC, 2010.

14 Aust DE, Baretton GB: Serrated polyps of the colon and rectum (hyperplastic polyps, sessile serrated adenomas, traditional serrated adenomas, and mixed polyps) - proposal for diagnostic criteria. Virchows Arch 2010;457:291-297.

15 Tannapfel A, Neid M, Aust D, Baretton G: The origins of colorectal carcinoma: specific nomenclature for different pathways and precursor lesions. Dtsch Arztebl Int 2010;107:760-766.

16 Morson BC: Precancerous lesions of the colon and rectum. Classification and controversial issues. JAMA 1962;179:316-321.

17 Lash RH, Genta RM, Schuler CM: Sessile serrated adenomas: prevalence of dysplasia and carcinoma in 2139 patients. J Clin Pathol 2010;63:681-686.

18 Lu FI, van Niekerk de W, Owen D, Tha SP, Turbin DA, Webber DL: Longitudinal outcome study of sessile serrated adenomas of the colorectum: an increased risk for subsequent right-sided colorectal carcinoma. Am J Surg Pathol;34:927-934.

19 Schmiegel W, Reinacher-Schick A, Arnold D, Graeven U, Heinemann V, Porschen R, Riemann J, Rodel C, Sauer R, Wieser M, Schmitt W, Schmoll HJ, Seufferlein T, Kopp I, Pox C: Update S3-guideline «Colorectal cancer» 2008. Z Gastroenterol 2008;46:799-840.
20 Di Nicolantonio F, Martini M, Molinari F, SartoreBianchi A, Arena S, Saletti P, De Dosso S, Mazzucchelli L, Frattini M, Siena S, Bardelli A: Wildtype $\mathrm{BRAF}$ is required for response to panitumumab or cetuximab in metastatic colorectal cancer. $\mathrm{J}$ Clin Oncol 2008:26:5705-5712.

21 Patel R, Shervington L, Lea R, Shervington A Epigenetic silencing of telomerase and a nonalkylating agent as a novel therapeutic approach for glioma. Brain Res 2008;1188:173-181.

22 Mossman D, Kim KT, Scott RJ: Demethylation by 5-aza-2'-deoxycytidine in colorectal cancer cells targets genomic DNA whilst promoter $\mathrm{CpG}$ island methylation persists. BMC Cancer 2010;10:366.

23 Dietmaier W: Microsatellite instability. A new predictive marker (?). Pathologe 2010;31(suppl 2): 268-273.

24 Amado RG, Wolf M, Peeters M, Van Cutsem E, Siena S, Freeman DJ, Juan T, Sikorski R, Suggs S, Radinsky R, Patterson SD, Chang DD: Wild-type KRAS is required for panitumumab efficacy in patients with metastatic colorectal cancer. J Clin Oncol 2008;26:1626-1634.

25 Bennecke M, Kriegl L, Bajbouj M, Retzlaff K, Robine S, Jung A, Arkan MC, Kirchner T, Greten FR: Ink4a/Arf and oncogene-induced senescence prevent tumor progression during alternative colorectal tumorigenesis. Cancer Cell 2010;18: 135-146.

26 Winawer SJ: The achievements, impact, and future of the national polyp study. Gastrointest Endosc 2006;64:975-978. 